\title{
Qualidade de um Argissolo submetido a práticas de manejo recuperadoras de sua estrutura física
}

\author{
Letícia Sequinatto ${ }^{1}$, Renato Levien², Carlos R. Trein ${ }^{3}$, Michael Mazurana ${ }^{4}$ \& Jônatan Müller ${ }^{5}$ \\ ${ }^{1}$ CAV/UDESC. Lages, SC. E-mail: letisequinatto@gmail.com (Autor correspondente) \\ ${ }^{2}$ Faculdade de Agronomia, UFRGS. Porto Alegre, RS. E-mail: renatole@ufrgs.br \\ ${ }^{3}$ Faculdade de Agronomia, UFRGS. Porto Alegre, RS. E-mail: trein@ufrgs.br \\ ${ }^{4}$ Faculdade de Agronomia, UFRGS. Porto Alegre, RS. E-mail: michael.mazurana@gmail.com \\ ${ }^{5}$ IFSC. São Miguel do Oeste, SC. Email: jonatan.muller@ifsc.edu.br
}

\section{Palavras-chave:}

compactação do solo

plantas de cobertura

tráfego controlado

matéria orgânica

\begin{abstract}
R E S U M O
Neste trabalho propôs-se avaliar a eficiência de alternativas de manejo na recuperação de um solo fisicamente degradado após oito anos. Para isto, um experimento manejado com semeadura direta vem sendo conduzido desde 2002, em Eldorado do Sul, RS, em um Argissolo Vermelho que, inicialmente, era campo natural degradado, com elevado grau de compactação. Os tratamentos englobaram três coberturas do solo no inverno (pousio, aveia preta + ervilhaca e nabo forrageiro), duas profundidades de atuação da haste sulcadora de adubo da semeadora $(0,12$ e $0,21 \mathrm{~m})$ e três condições de tráfego (tráfego de rodados de trator, colhedora e sem tráfego). No verão são semeados milho e soja em rotação anual. Constatou-se aumento nos teores de matéria orgânica do solo e de suas frações, diminuição dos valores de densidade e aumento da macroporosidade e da porosidade total do solo. A densidade do solo foi menor na condição de solo não trafegado por rodados de máquinas agrícolas. A conversão do campo natural degradado para um sistema de produção de grãos utilizando alternativas integradas de manejo com solo em semeadura direta reestrutura o solo, atingindo qualidade física para desempenhar adequadamente suas funções.
\end{abstract}

Key words: soil compaction cover crops controlled traffic organic matter

\section{Quality of an Ultisol subjected to management practices to reclaim physical structure}

\begin{abstract}
A B S T R A C T
The aim of this study was to evaluate the effectiveness of alternative managements in restoring a physically degraded soil after eight years. For this, an experiment managed with direct sowing (no tillage) is being conducted since 2002, in Eldorado do Sul, RS, in an Ultisol that initially was degraded naturally with a high degree of compaction. Treatments consisted of three soil cover crops in winter (fallow, oat + vetch and forage radish), two depths $(0.12$ and $0.21 \mathrm{~m})$ of operation of the shank of fertilizer and seed drill and three traffic conditions (tractor movements, harvester and no traffic). In summer corn and soybeans are sown in annual rotation. There was an increase in the levels of soil organic matter and of its fractions, which resulted in reduced values of density and increased macroporosity and total porosity of soil. Soil bulk density was lower in soil condition not without agricultural machinery traffic. The conversion of naturally degraded field into a system of grain production using integrated management alternatives with no tillage system restores the physical quality of soil to carry out appropriately its functions.
\end{abstract}

\section{INTRODUÇÃO}

A compactação degrada a estrutura física dos solos e tem reflexos negativos sobre o desempenho adequado de suas funções (Karlen et al., 1997), especialmente sua capacidade de servir como meio de crescimento e desenvolvimento das plantas (Reichert et al., 2003). Este fato ocorre porque a compactação aumenta a densidade do solo (Ds), que afeta a quantidade, tamanho, tortuosidade e continuidade dos poros no solo interferindo sobre o armazenamento, transporte e disponibilidade de água às plantas e no fluxo de gases (Peth \& Horn, 2006).
A recuperação física de solos degradados buscando qualidade para desempenhar suas funções é alcançada através de práticas de manejo integradas do sistema agrícola (Fliebbach et al., 2007). Ao longo do tempo a semeadura direta (SD) melhora a estrutura do solo; entretanto, o tráfego de máquinas e o baixo revolvimento do solo podem resultar em níveis de compactação prejudiciais ao desenvolvimento de plantas. Sistemas de rotação de culturas em SD que incluam plantas de cobertura de inverno melhoram a estrutura do solo (Hamza \& Anderson, 2005), sobretudo mediante a abertura de bioporos pelo sistema radicular (Dexter, 1991) e aumento do teor de matéria orgânica do solo (MOS) (Oades, 1993). A MOS reduz 
a Ds (Jarecki et al., 2005; Braida, et al., 2008), fato creditado ao seu efeito sobre a agregação, menor densidade de partícula e baixo grau de empacotamento (Soane, 1990).

A eficiência das plantas de cobertura em recuperar fisicamente os solos e diminuir o efeito do tráfego agrícola na compactação pode ser melhorada se for complementada pela limitação do tráfego a determinadas regiões, dentro da lavoura (tráfego controlado) e pelo uso de hastes sulcadoras da semeadora mobilizando o solo em maiores profundidades; mencionadas práticas diminuem o grau de compactação (Veiga et al., 2007; Debiasi et al., 2008). No entanto, o período de tempo durante o qual um solo degradado leva para adquirir estrutura estável e qualidade para a sustentabilidade agrícola, ainda é pouco conhecido.

Objetivou-se nesta pesquisa avaliar o efeito de práticas integradas de manejo do solo sob SD, como a utilização de plantas de cobertura de inverno, do tráfego controlado e do grau de mobilização na linha de semeadura das culturas de verão sobre algumas propriedades físicas de um Argissolo em processo de recuperação.

\section{MATERIAL E MÉTOdos}

O experimento se localiza na Estação Experimental Agronômica da Universidade Federal do Rio Grande do Sul, no município de Eldorado do Sul, RS. O solo é classificado como Argissolo Vermelho distrófico típico (EMBRAPA, 2006), de textura superficial franco-argilo-arenosa, com teores de argila, silte e areia de 270, 220 e $510 \mathrm{~g} \mathrm{~kg}^{-1}$ (Beutler, 2005).

O solo da área possui histórico de degradação estrutural intensa; na década de 70 era preparado com o uso de aração, gradagem e enxada rotativa, para pesquisas com melhoramento de cultivares, ao final das quais ocorreu o restabelecimento de espécies de plantas dos campos naturais da região, principalmente gramíneas e, em 1982, a área passou a ser utilizada como área de confinamento semi-intensivo de bovinos, até 2002, quando se iniciou a presente pesquisa. Avaliações físicas realizadas naquele ano indicaram que o solo possuía alta Ds (1,71 $\left.\mathrm{Mg} \mathrm{m}^{-3}\right)$ e baixas macroporosidade (MAC) $(0,06$ $\left.\mathrm{m}^{3} \mathrm{~m}^{-3}\right)$ e porosidade total $(\mathrm{Pt})\left(0,35 \mathrm{~m}^{3} \mathrm{~m}^{-3}\right)$ no perfil até 0,12 $\mathrm{m}$ de profundidade (Beutler, 2005), caracterizando um solo fisicamente degradado pela compactação. A conversão da área para uso com culturas anuais em SD foi realizada sem o revolvimento do solo.

O delineamento experimental utilizado foi o de blocos ao acaso, com parcelas subsubdivididas e quatro repetições. Os tratamentos principais, dispostos em parcelas de $4,5 \mathrm{~m}$ de largura por $20 \mathrm{~m}$ de comprimento, constaram de três coberturas de inverno (pousio - P; nabo forrageiro - NF e consórcio aveia preta + ervilhaca - AE), seguidas da semeadura de milho e soja no verão, em rotação anual. Nas áreas sob $\mathrm{P}$ as espécies vegetais predominantes com restabelecimento natural durante o inverno, foram a serralha, o azevém, o mentruz e a gramaseda. O espaçamento utilizado na semeadura das culturas de cobertura de solo no inverno e nas produtoras de grãos de verão foi respectivamente de 0,17 e 0,45 m enquanto nas subparcelas os tratamentos foram profundidade de atuação da haste sulcadora de adubo da semeadora $(0,12$ e $0,21 \mathrm{~m})$ e nas subsubparcelas o tráfego controlado (áreas não trafegadas; áreas trafegadas somente por rodados do trator e áreas trafegadas apenas por rodados da colhedora).

Foram analisadas as propriedades físicas Ds e porosidade da mesma forma que a MOS (frações particulada - MOP e associada aos minerais - MOM), nos anos de 2008 e 2009. Os resultados foram comparados com dados obtidos anteriormente por Beutler (2005) e Debiasi (2008) que utilizaram a área experimental com os mesmos tratamentos.

A coleta de amostras indeformadas de solo para determinação das Ds, Pt, MAC e microporosidade (MIC) foi realizada em dezembro de 2008 nos locais com tráfego de rodado do trator (RT), tráfego de rodado da colhedora (RC) e sem tráfego de rodados (NT). Nesses locais foram coletadas três amostras de solo nas camadas de 0,02-0,05 m, 0,10-0,13 m e 0,20-0,23 m, com aneis volumétricos de $0,055 \mathrm{~m}$ de diâmetro e $0,03 \mathrm{~m}$ de altura. Em laboratório as amostras foram saturadas em água destilada, durante $48 \mathrm{~h}$, pesadas e equilibradas na tensão de $6 \mathrm{kPa}$; em seguida, foram colocadas em estufa a $105^{\circ} \mathrm{C}$ e aí mantidas até atingirem peso constante $(72 \mathrm{~h})$; enfim, as determinações das Ds, da MAC, da MIC e do Pt do solo foram realizadas segundo EMBRAPA (1997).

Visando à determinação dos teores de MOS, MOP e MOM foram coletadas, em junho de 2009, três amostras de solo em cada parcela nas camadas de 0,02 a $0,05 \mathrm{~m}, 0,10$ a $0,13 \mathrm{~m}$ e 0,20 a $0,23 \mathrm{~m}$ e analisadas segundo o método de Walkley-Black modificado (Tedesco et al., 1995).

A determinação da matéria seca (MS) das culturas de cobertura de inverno e do pousio foi obtida pelo corte rente ao solo de todas as plantas contidas no interior de um retângulo $\left(0,24 \mathrm{~m}^{2}\right)$, em todas as parcelas. As amostras foram secas em estufa, na temperatura de $65^{\circ} \mathrm{C}$ até peso constante, sendo os valores assim obtidos extrapolados para $\mathrm{Mg} \mathrm{ha}^{-1}$.

A análise da variância (ANOVA) e os testes de comparação entre as médias foram realizados pelo teste Tukey a 0,05 de significância, com auxílio do programa computacional SAS (SAS Institute, 2002). Para os parâmetros físicos Ds, Pt, MAC, MIC e teores de MOS, MOP e MOM o modelo utilizado para a ANOVA foi o bifatorial. Quando da ocorrência de interações significativas (teste $\mathrm{F}, \mathrm{p}<0,05$ ) as médias dos níveis de um fator foram comparadas dentro de outro.

\section{Resultados e Discussão}

Nas Tabelas 1, 2 e 3 são apresentadas as médias dos teores de MOS e suas frações tal como também a produção de matéria seca das culturas de cobertura de inverno.

As plantas de cobertura de solo no inverno resultaram em maior teor de MOS na superfície do solo $(0,02-0,05 \mathrm{~m})$ comparativamente ao pousio (Tabela 1) evento passível de ser 
Tabela 1. Teor de matéria orgânica do solo (MOS), matéria orgânica particulada (MOP) e matéria orgânica associada aos minerais (MOM) em função da cultura de cobertura de solo de inverno (aveia preta + ervilhaca - $A E$; nabo forrageiro - NF; pousio - $\mathrm{P}$ ), da cultura de verão antecessora à amostragem e da camada de avaliação (Junho/2009)

\begin{tabular}{|c|c|c|c|c|c|}
\hline \multirow{2}{*}{$\begin{array}{c}\text { Camada } \\
\text { (m) }\end{array}$} & \multirow{2}{*}{$\begin{array}{c}\text { Cultura } \\
\text { de verão }\end{array}$} & \multicolumn{3}{|c|}{ Cobertura de inverno } & \multirow{2}{*}{ Média } \\
\hline & & $\mathrm{AE}$ & NF & $\mathbf{P}$ & \\
\hline & & \multicolumn{4}{|c|}{$\operatorname{MOS}\left(\mathrm{g} \mathrm{kg}^{-1}\right)$} \\
\hline \multirow{4}{*}{$0,02-0,05$} & Milho & 40,99 & 41,07 & 32,25 & $38,11 \mathrm{~B}$ \\
\hline & Soja & 44,00 & 53,57 & 45,02 & $47,53 \mathrm{~A}$ \\
\hline & Média & $42,50 a b$ & $47,32 \mathrm{a}$ & $38,63 \mathrm{~b}$ & \\
\hline & Milho & 22,94 & 20,09 & 23,10 & $22,04 \mathrm{~A}$ \\
\hline \multirow[t]{3}{*}{$0,10-0,13$} & Soja & 22,23 & 22,13 & 25,18 & $23,18 \mathrm{~A}$ \\
\hline & Média & $22,58 a b$ & $21,11 \mathrm{~b}$ & $24,14 a$ & \\
\hline & Milho & 17,95 & 18,32 & 16,28 & $17,52 \mathrm{~A}$ \\
\hline \multirow[t]{4}{*}{$0,20-0,23$} & Soja & 16,24 & 16,40 & 18,31 & $16,98 \mathrm{~A}$ \\
\hline & Média & $17,09 \mathrm{a}$ & $17,36 \mathrm{a}$ & $17,29 \mathrm{a}$ & \\
\hline & & \multicolumn{4}{|c|}{ MOP $\left(\mathrm{g} \mathrm{kg}^{-1}\right)$} \\
\hline & Milho & 9,59 & 9,41 & 9,23 & $9,41 \mathrm{~A}$ \\
\hline \multirow[t]{3}{*}{$0,02-0,05$} & Soja & 9,80 & 12,34 & 9,68 & $10,61 \mathrm{~A}$ \\
\hline & Média & $9,69 a$ & $10,88 \mathrm{a}$ & $9,45 \mathrm{a}$ & \\
\hline & Milho & $1,77 a b$ & $1,19 b$ & $2,26 \mathrm{a}$ & $1,74 \mathrm{~A}$ \\
\hline \multirow[t]{3}{*}{$0,10-0,13$} & Soja & $1,30 \mathrm{~b}$ & $1,97 \mathrm{~b}$ & $2,99 \mathrm{a}$ & $2,09 \mathrm{~A}$ \\
\hline & Média & $1,53 \mathrm{~b}$ & $1,58 a b$ & $2,63 \mathrm{a}$ & \\
\hline & Milho & 1,03 & 1,05 & 0,57 & $0,88 \mathrm{~A}$ \\
\hline \multirow[t]{4}{*}{$0,20-0,23$} & Soja & 0,81 & 0,56 & 0,95 & $0,77 \mathrm{~A}$ \\
\hline & Média & $0,93 \mathrm{a}$ & $0,81 \mathrm{a}$ & $0,76 \mathrm{a}$ & \\
\hline & & \multicolumn{4}{|c|}{ MOM $\left(\mathrm{g} \mathrm{kg}^{-1}\right)$} \\
\hline & Milho & $31,41 \mathrm{~A}$ & $31,66 \mathrm{~B}$ & 23,02 B & $28,70 \mathrm{~A}$ \\
\hline \multirow[t]{3}{*}{$0,02-0,05$} & Soja & $34,20 \mathrm{~A}$ & $41,22 \mathrm{~A}$ & $35,34 \mathrm{~A}$ & $36,92 \mathrm{~A}$ \\
\hline & Média & $32,81 a b$ & $36,44 a$ & $29,18 \mathrm{~b}$ & \\
\hline & Milho & 21,17 & 18,90 & 20,83 & $20,30 \mathrm{~A}$ \\
\hline \multirow[t]{3}{*}{$0,10-0,13$} & Soja & 20,93 & 20,17 & 22,18 & $21,09 \mathrm{~A}$ \\
\hline & Média & $21,05 a b$ & $19,53 \mathrm{~b}$ & $21,51 \mathrm{a}$ & \\
\hline & Milho & 16,91 & 17,28 & 15,70 & $16,63 \mathrm{~A}$ \\
\hline \multirow[t]{2}{*}{$0,20-0,23$} & Soja & 15,42 & 15,83 & 17,36 & $16,20 \mathrm{~A}$ \\
\hline & Média & $16,16 \mathrm{a}$ & $16,55 \mathrm{a}$ & $16,53 \mathrm{a}$ & \\
\hline
\end{tabular}

Médias seguidas pela mesma letra maiúscula nas colunas e minúscula nas linhas, dentro de cada camada, não diferem significativamente entre si, pelo teste de Tukey $(p<0,05)$
Tabela 2. Produção de massa seca da parte aérea $\left(\mathrm{Mg} \mathrm{ha}^{-1}\right)$ das culturas de cobertura de solo no inverno

\begin{tabular}{lcccc}
\hline \multicolumn{1}{c}{ Cobertura do solo } & \multicolumn{4}{c}{ Ano $^{1}$} \\
\cline { 2 - 5 } no inverno & $\mathbf{2 0 0 5}$ & $\mathbf{2 0 0 6}$ & $\mathbf{2 0 0 8}$ & $\mathbf{2 0 0 9}$ \\
Nabo forrageiro & $11,0 \mathrm{a}$ & $10,3 \mathrm{a}$ & $8,37 \mathrm{a}$ & $9,45 \mathrm{a}$ \\
Aveia preta ou aveia & $8,6 \mathrm{~b}$ & $7,1 \mathrm{~b}$ & $5,02 \mathrm{ab}$ & $8,12 \mathrm{a}$ \\
preta + ervilhaca ${ }^{2}$ & & & & \\
Pousio & $4,5 \mathrm{c}$ & $2,4 \mathrm{c}$ & $3,32 \mathrm{~b}$ & $4,50 \mathrm{~b}$ \\
CV (\%) & 15,1 & 20,8 & 18,10 & 17,90 \\
\hline
\end{tabular}

Médias seguidas pela mesma letra minúscula nas colunas não diferem significativamente entre si pelo teste de Tukey $(p<0,05)$.

${ }^{1}$ Os dados referentes aos anos de 2005 e 2006 foram obtidos por Debiasi (2008).

${ }^{2}$ Em 2007 foi consorciada a ervilhaca com a aveia preta.

atribuído à maior adição de resíduos provenientes da parte aérea das culturas de cobertura (Tabela 2) e ao aporte de material orgânico via sistema radicular visto que, de acordo com Beutler (2005) e Souza et al. (2009), há uma proporcionalidade entre a massa da parte aérea e raízes das plantas de cobertura.

No ano de 2006 Debiasi (2008), observou que as plantas de cobertura de inverno foram responsáveis por uma quantidade maior de MOS na camada superficial com valor médio de 36,95 $\mathrm{g} \mathrm{kg}^{-1}$ (Tabela 3), devido à elevada MS (Tabela 2), porém este valor de MOS foi menor que o obtido no ano de 2009, de 44,91 $\mathrm{g} \mathrm{kg}^{-1}$ (Tabela 3), para a camada superficial, o que se aproximou dos valores encontrados para o campo nativo (CN) $(46,90 \mathrm{~g}$ $\mathrm{kg}^{-1}$ ), indicando aumento de $18 \%$ em três anos. O P também apresentou aumento nos teores de MOS na camada superficial passando de $31,80 \mathrm{~g} \mathrm{~kg}^{-1}$, em 2006, a 38,63 $\mathrm{g} \mathrm{kg}^{-1}$, em 2009, ou seja, incremento de aproximadamente $18 \%$ (Tabela 3).

A maior adição de MOS pelas plantas de cobertura de solo na camada superficial está sendo influenciada, em maiores proporções, pelo sistema com NF (Tabela 1). Este possui maior velocidade inicial de acúmulo de matéria verde e seca em relação à aveia preta e à ervilhaca comum (Balbinot Junior et al., 2004). Isto, aliado ao fato de ter apresentado, nos dois anos, maior quantidade de MS (Tabela 2), deve ter favorecido o maior incremento nos teores de MOS.

Tabela 3. Teores de matéria orgânica do solo (MOS), matéria orgânica particulada (MOP) e matéria orgânica associada aos minerais (MOM) em função da condição de cobertura de solo de inverno, em junho dos anos de 2006 e de 2009

\begin{tabular}{|c|c|c|c|c|c|c|c|}
\hline \multirow{3}{*}{$\begin{array}{c}\text { Camada }{ }^{1} \\
\text { (m) }\end{array}$} & \multirow{3}{*}{ Cobertura de inverno ${ }^{2}$} & \multicolumn{6}{|c|}{ Matéria orgânica $\left(\mathrm{g} \mathrm{kg}^{-1}\right)$} \\
\hline & & \multicolumn{2}{|c|}{ MOS } & \multicolumn{2}{|c|}{ MOP } & \multicolumn{2}{|c|}{ MOM } \\
\hline & & 2006 & 2009 & 2006 & 2009 & 2006 & 2009 \\
\hline \multirow{6}{*}{$\begin{array}{c}0,03-0,06 \\
\text { ou } \\
0,02-0,05\end{array}$} & AE ou NF & $35,70 \mathrm{a}$ & $47,32 \mathrm{a}$ & $6,30 \mathrm{~b}$ & $10,88 \mathrm{a}$ & $29,40 \mathrm{a}$ & $36,44 a$ \\
\hline & A ou $\mathrm{AE}$ & $38,20 \mathrm{a}$ & $42,50 \mathrm{ab}$ & $7,90 \mathrm{a}$ & $9,69 a$ & $30,30 \mathrm{a}$ & $32,81 \mathrm{a}$ \\
\hline & $P$ & $31,80 \mathrm{a}$ & $38,63 \mathrm{~b}$ & $3,70 \mathrm{~b}$ & $9,45 \mathrm{a}$ & $28,10 \mathrm{a}$ & $29,18 b$ \\
\hline & Média 1 & 36,95 & 44,91 & 7,10 & 10,29 & 29,85 & 34,63 \\
\hline & Média 2 & 35,23 & 42,81 & 6,00 & 10,00 & 29,30 & 32,81 \\
\hline & $\mathrm{CN}$ & 46,90 & & 12,60 & & 34,30 & \\
\hline \multirow{6}{*}{$\begin{array}{c}0,10-0,13 \\
\text { ou } \\
0,12-0,15\end{array}$} & AE ou NF & $18,80 \mathrm{a}$ & $21,11 b$ & $1,40 \mathrm{a}$ & $1,58 \mathrm{ab}$ & $17,40 \mathrm{a}$ & $19,53 \mathrm{~b}$ \\
\hline & A ou $A E$ & $18,90 \mathrm{a}$ & $22,58 a b$ & $0,90 \mathrm{a}$ & $1,53 \mathrm{~b}$ & $18,00 \mathrm{a}$ & $21,05 a b$ \\
\hline & $P$ & $18,20 \mathrm{a}$ & $24,14 \mathrm{a}$ & $1,00 \mathrm{a}$ & $2,63 \mathrm{a}$ & $17,20 \mathrm{a}$ & $21,51 \mathrm{a}$ \\
\hline & Média 1 & 18,85 & 21,85 & 1,15 & 1,55 & 17,70 & 20,59 \\
\hline & Média 2 & 18,63 & 22,61 & 1,10 & 1,91 & 17,53 & 20,70 \\
\hline & $\mathrm{CN}$ & 25,20 & & 3,30 & & 21,90 & \\
\hline
\end{tabular}

Médias seguidas da mesma letra minúscula nas colunas dentro de cada camada e ano de avaliação não diferem significativamente entre si, pelo teste de Tukey ( $p<0,05)$.

${ }^{1}$ Em 2006 as avaliações realizadas por Debiasi (2008), foram determinadas nas camadas de 0,03-0,06 m e 0,12-0,15 m, e, em 2009 nas camadas de 0,02-0,05 m e 0,12-0,15 m.

${ }^{2}$ AE - Consórcio aveia preta + ervilhaca; NF - Nabo forrageiro; A - Aveia; P - Pousio; CN - Campo nativo; Média 1 - Culturas de coberturas de inverno; Média 2 - Todas as coberturas de inverno. No ano de 2006 a AE foi substituída por NF e em 2007 a AE foi substituída por AE 
O teor de MOS foi influenciado de maneira significativa pela cultura de verão na camada de 0,02-0,05 m (Tabela 1). Foi maior a quantidade de MOS quando a cultura antecessora à amostragem foi a soja em virtude da elevada taxa de decomposição dos seus resíduos.

Na camada de 0,10-0,13 mo teor de MOS foi significativamente inferior ao de 0,02-0,05 m, em aproximadamente 50\%, tendo sido afetado pelas coberturas de inverno (Tabela 1). Em estudos em manejo do solo com a SD, Conceição (2006) indica que a magnitude do incremento no teor de MOS, ocasionado pelo emprego de plantas de cobertura, é reduzida com o aumento da profundidade da camada avaliada. Analisando a Tabela 3 verifica-se que houve incremento de MOS na segunda camada do ano 2006 para 2009, em aproximadamente $18 \%$, ou seja, na mesma proporção da MOS para a camada superficial.

Os sistemas $\mathrm{P}$ e AE apresentaram os maiores teores de MOS na camada de $0,10-0,13 \mathrm{~m}$ (Tabela 1), respectivamente de 24,14 e 22,58 $\mathrm{g} \mathrm{kg}^{-1}$, aproximando-se ao CN $\left(25,20 \mathrm{~g} \mathrm{~kg}^{-1}\right.$ ) (Tabela 3). Na mesma camada constatou-se incremento nos teores de MOS dos anos 2006 a 2008, em aproximadamente 18\% (Tabela 3). O que pode estar contribuindo para o acúmulo de MOS em profundidade é o sistema radicular em decomposição das plantas e, como salientado por Souza et al. (2009), o transporte de resíduos vegetais da superfície do solo para as camadas mais profundas, pela macro e mesofauna do solo. O incremento nos teores de MOS no P para a camada de 0,10-0,13 m é reflexo da recuperação acentuada da estrutura do solo. Anualmente, durante os anos de manejo o $\mathrm{P}$ sofreu menos mobilização do solo excetuando-se o plantio das culturas de verão, o que mantém a estrutura do solo inalterada por mais tempo, em comparação com os sistemas de manejo que necessitam do plantio das culturas de inverno. Por outro lado, apresenta alto restabelecimento natural de espécies de inverno sobremaneira de gramíneas, caracteristicamente com sistema radicular denso. Portanto, o manejo do solo no sistema em P pode ter promovido o alcance do equilíbrio estrutural. Esse estado estável é alcançado em resposta às práticas de manejo do solo no tempo, quando há um balanço constante entre a magnitude dos processos que resultam na degradação e aqueles que proporcionam a construção da estrutura (Vezzani \& Mielniczuk, 2011).

Em 2009 e na camada de 0,20-023 m o teor médio de MOS, de $17,25 \mathrm{~g} \mathrm{~kg}^{-1}$ para todos os sistemas (Tabela 1), assemelhou-se aos encontrados em 2006 para a camada de 0,12-0,15 m, que foi de $18,63 \mathrm{~g} \mathrm{~kg}^{-1}$ (Tabela 3). Isto prova o incremento de MOS em subsuperfície no decorrer do tempo. Diekow et al. (2005) citam haver, em geral, um limite de acúmulo de MOS para os solos. Bayer et al. (2000) concluíram, através de simulações baseadas em dados obtidos em um experimento de longa duração, que sob adições de $\mathrm{C}$ semelhantes às encontradas nos sistemas de rotação incluindo plantas de cobertura de inverno, a concentração de equilíbrio da MOS na camada de $0,0-0,175$ m poderá chegar a aproximadamente $42,0 \mathrm{~g} \mathrm{~kg}^{-1}$.

O teor de MOP na camada de 0,02-0,05 $\mathrm{m}$ foi semelhante entre os sistemas de manejo incluindo as plantas de cobertura de solo de inverno (Tabela 1); entretanto, houve tendência de se apresentar maior nos sistemas de manejo com as plantas de cobertura de inverno estando de acordo, então, com os teores de MOS na mesma camada.

Na camada de 0,10-0,13 m as plantas de cobertura de inverno afetaram os valores de MOP para uma mesma cultura de verão (Tabela 1). Quando a cultura de verão antecessora à amostragem foi o milho, a cobertura de inverno que apresentou o maior teor de MOP foi o $P\left(2,26 \mathrm{~g} \mathrm{~kg}^{-1}\right)$, não diferindo estatisticamente do $\mathrm{AE}\left(1,77 \mathrm{~g} \mathrm{~kg}^{-1}\right)$ mas quando a cultura de verão foi a soja novamente o maior teor de MOP foi do sistema em P (2,99 $\mathrm{g}$ $\left.\mathrm{kg}^{-1}\right)$.

A Tabela 3 evidencia o aumento pronunciado nos teores de MOP entre os anos 2006 e 2009, para todos os sistemas de cobertura do solo nas duas camadas, em torno de $40 \%$ sendo este valor maior que os $18 \%$ de aumento de MOS no período, demonstrando que a fração lábil da MOS foi mais sensível às mudanças do manejo que a MOS, confirmando as conclusões já obtidas por Conceição et al. (2005) e Debiasi (2008). Bayer et al. (2004) ao citar que há maior sensibilidade do estoque de $\mathrm{C}$ na MOP em virtude das alterações no manejo.

Os teores de MOM foram afetados pelos sistemas de cobertura de inverno de solo nas duas primeiras camadas de solo avaliadas (Tabela 1). As adições de resíduos proporcionadas pelas culturas no tempo implicaram sobre o aumento nos teores de MOM, também detectado por Conceição (2006), em um experimento de longa duração.

As diferentes profundidades de atuação das hastes sulcadoras não influenciaram a Ds nem a porosidade do solo dentro das camadas e manejos do solo avaliados (dados não apresentados) (Sequinatto, 2010) provavelmente em função do local de coleta das amostras de solo ter ocorrido fora da linha de semeadura. Entretanto, a maior mobilização do solo pelas hastes sulcadoras pode influenciar a conservação da água no sistema solo semelhante aos sistemas com solos escarificados esporadicamente, como salientado por Câmara \& Klein (2005).

Houve interação significativa entre as coberturas de solo de inverno e a condição de tráfego para a Ds, nas camadas de 0,02-0,05 m (Tabela 4). Dentro dos sistemas com NTD o solo apresentou as menores Ds $\left(1,09 \mathrm{Mg} \mathrm{m}^{-3}\right)$ embora se tenha igualado ao RT (1,30 $\left.\mathrm{Mg} \mathrm{m}^{-3}\right)$.

$\mathrm{Na}$ camada superficial (Tabela 4), as regiões de solo que não receberam tráfego apresentaram a menor Ds $(1,19 \mathrm{Mg}$ $\mathrm{m}^{-3}$ ) enquanto no solo trafegado por trator ou colhedora a Ds foi de 1,33 e 1,36 $\mathrm{Mg} \mathrm{m}^{-3}$, respectivamente. Esses valores estão abaixo dos valores de Ds críticos estabelecidos por Reichert et al. (2003) que, para a classe textural do solo desta pesquisa, é em torno de $1,45 \mathrm{Mg} \mathrm{m}^{-3}$. Em avaliações no ano de 2006, Debiasi et al. (2008) encontraram, nas regiões não trafegadas e trafegadas, respectivamente, valores de Ds em torno de 1,34 e $1,43 \mathrm{Mg} \mathrm{m}^{-3}$ indicando redução significativa nos valores de Ds, relacionada principalmente ao tempo de manejo de solo com as plantas de cobertura.

$\mathrm{Na}$ camada de 0,10-0,13 m a Ds foi influenciada pela interação entre RC e as culturas de cobertura de inverno (Tabela 4). O RC apresentou a maior Ds quando a cobertura de inverno 
Tabela 4. Densidade do solo (Ds) em função da condição de cobertura de solo de inverno (aveia preta + ervilhaca $-\mathrm{AE}$; nabo forrageiro - NF; pousio - P), do tráfego dos rodados (rodados de colhedora - RC; solo não trafegado - NT; rodados de trator - RT) e da camada de avaliação (dezembro, 2008)

\begin{tabular}{|c|c|c|c|c|c|}
\hline \multirow{3}{*}{$\begin{array}{c}\text { Camada } \\
(\mathrm{m})\end{array}$} & \multirow{3}{*}{ Tráfego } & \multicolumn{3}{|c|}{ Cobertura de inverno } & \multirow{2}{*}{ Média } \\
\hline & & $\mathrm{AE}$ & NF & $P$ & \\
\hline & & \multicolumn{4}{|c|}{ Ds $\left(\mathrm{Mg} \mathrm{m}^{-3}\right)$} \\
\hline \multirow{4}{*}{$0,02-0,05$} & $\mathrm{RC}$ & $1,32 \mathrm{~A}$ & $1,40 \mathrm{~A}$ & $1,36 \mathrm{~A}$ & $1,36 \mathrm{~A}$ \\
\hline & NT & $1,24 \mathrm{~A}$ & $1,09 \mathrm{~B}$ & $1,25 \mathrm{~A}$ & $1,19 \mathrm{~B}$ \\
\hline & RT & $1,36 \mathrm{~A}$ & $1,30 \mathrm{AB}$ & $1,33 \mathrm{~A}$ & $1,33 \mathrm{~A}$ \\
\hline & Média & $1,31 \mathrm{a}$ & $1,26 \mathrm{a}$ & $1,31 \mathrm{a}$ & \\
\hline \multirow{4}{*}{$0,10-0,13$} & $\mathrm{RC}$ & $1,71 \mathrm{a}$ & $1,60 a b$ & $1,49 b$ & $1,60 \mathrm{~A}$ \\
\hline & NT & $1,55 \mathrm{a}$ & $1,44 \mathrm{a}$ & $1,51 \mathrm{a}$ & $1,51 \mathrm{~B}$ \\
\hline & RT & $1,63 \mathrm{a}$ & $1,62 a$ & $1,51 \mathrm{a}$ & $1,59 \mathrm{AB}$ \\
\hline & Média & $1,63 \mathrm{a}$ & $1,55 a b$ & $1,51 \mathrm{~b}$ & \\
\hline \multirow{4}{*}{$0,20-0,23$} & $\mathrm{RC}$ & 1,70 & 1,69 & 1,60 & $1,66 \mathrm{~A}$ \\
\hline & NT & 1,62 & 1,67 & 1,67 & $1,65 \mathrm{~A}$ \\
\hline & RT & 1,61 & 1,68 & 1,67 & $1,65 \mathrm{~A}$ \\
\hline & Média & $1,64 \mathrm{a}$ & $1,68 \mathrm{a}$ & $1,64 \mathrm{a}$ & \\
\hline
\end{tabular}

Médias seguidas pela mesma letra maiúscula nas colunas e minúscula nas linhas, dentro de cada camada, não diferem significativamente entre si, pelo teste de Tukey $(p<0,05)$

foi $\operatorname{AE}\left(1,71 \mathrm{Mg} \mathrm{m}^{-3}\right)$, não diferindo do $\mathrm{NF}\left(1,60 \mathrm{Mg} \mathrm{m}^{-3}\right)$; na média, os maiores valores de Ds foram encontrados para RC e RT, respectivamente de 1,60 e $1,59 \mathrm{Mg} \mathrm{m}^{-3}$, diferindo do NT $\left(1,51 \mathrm{Mg} \mathrm{m}^{-3}\right)$. Em 2006 a Ds no solo NT e trafegado foi de 1,59 e 1,60 $\mathrm{Mg} \mathrm{m}^{-3}$, respectivamente (Debiasi et al., 2008), indicando redução dos valores de Ds somente para as regiões de solo NT, em dois anos.

A cobertura de solo no inverno influenciou os valores de Ds para a camada de 0,10 - 0,13 m (Tabela 4). A menor Ds foi obtida no $\mathrm{P}\left(1,51 \mathrm{Mg} \mathrm{m}^{-3}\right)$, a qual não diferiu do NF $(1,55 \mathrm{Mg}$ $\mathrm{m}^{-3}$ ) de acordo com os teores de MOS e MOP (Tabela 1); seus incrementos resultaram em decréscimos da Ds. Maiores valores de Ds encontrados para a camada de 0,20-0,23 m em relação às camadas mais superficiais podem estar relacionados ao adensamento natural das partículas do solo não se constituindo reflexo das práticas de manejo nem do tráfego de rodados.

A condição de tráfego alterou a Pt (Tabela 5). Maiores valores de Pt foram obtidos, em se considerando as duas primeiras camadas avaliadas, para o NT, embora se tenham igualado estatisticamente às demais condições de tráfego. A camada de 0,02-0,05 $\mathrm{m}$ apresentou a maior Pt (Tabela 5) com valor médio de $0,57 \mathrm{~m}^{3} \mathrm{~m}^{-3}$ para o NT valor este maior que o encontrado por Debiasi et al. (2008), no ano de 2006, que foi de $0,48 \mathrm{~m}^{3} \mathrm{~m}^{-3}$, representando um incremento de aproximadamente $16 \%$, em dois anos. Em 2006 a Pt do solo com tráfego foi de $0,44 \mathrm{~m}^{3} \mathrm{~m}^{-3}$ e, portanto, menor do que os valores médios atuais $\left(0,53 \mathrm{~m}^{3} \mathrm{~m}^{-3}\right)$. Em 2002 a Pt encontrada por Beutler (2005) foi de $0,36 \mathrm{~m}^{3} \mathrm{~m}^{-3}$ com que se salienta que houve evolução da estrutura do solo no tempo.

$\mathrm{Na}$ camada de $0,10-0,13 \mathrm{~m}$ a Pt foi influenciada pela cobertura de solo no inverno de forma que os maiores valores foram encontrados para o P e para o NF (Tabela 5). A maior Pt foi obtida para o NT $\left(0,47 \mathrm{~m}^{3} \mathrm{~m}^{-3}\right)$, embora semelhante ao $\mathrm{RC}(0,44$ $\mathrm{m}^{3} \mathrm{~m}^{-3}$ ). Em 2006 Debiasi et al. (2008) encontraram valor de Pt
Tabela 5. Valores de porosidade total $(\mathrm{Pt})$, macroporosidade (MAC) e microporosidade (MIC) do solo em função da condição de cobertura de solo no inverno (aveia + ervilhaca - $\mathrm{AE}$; nabo forrageiro - NF; pousio - P) do tráfego dos rodados (rodados de colhedora - RC; solo não trafegado - NT; rodados de trator - RT) e da camada de avaliação (dezembro, 2008)

\begin{tabular}{|c|c|c|c|c|c|}
\hline \multirow{2}{*}{$\begin{array}{c}\text { Camada } \\
\text { (m) }\end{array}$} & \multirow{2}{*}{ Tráfego } & \multicolumn{3}{|c|}{ Cobertura de inverno } & \multirow{2}{*}{ Média } \\
\hline & & $\overline{A E}$ & NF & $\mathbf{P}$ & \\
\hline & & \multicolumn{4}{|c|}{$\mathrm{Pt}\left(\mathrm{m}^{3} \mathrm{~m}^{-3}\right)$} \\
\hline \multirow{4}{*}{$0,02-0,05$} & $\mathrm{RC}$ & 0,53 & 0,52 & 0,54 & $0,53 \mathrm{~B}$ \\
\hline & NT & 0,59 & 0,58 & 0,55 & $0,57 \mathrm{~A}$ \\
\hline & RT & 0,53 & 0,54 & 0,55 & $0,54 \mathrm{AB}$ \\
\hline & Média & $0,55 \mathrm{a}$ & $0,55 \mathrm{a}$ & $0,55 \mathrm{a}$ & \\
\hline \multirow{4}{*}{$0,10-0,13$} & $\mathrm{RC}$ & 0,40 & 0,44 & 0,48 & $0,44 \mathrm{AB}$ \\
\hline & NT & 0,46 & 0,50 & 0,46 & $0,47 \mathrm{~A}$ \\
\hline & RT & 0,40 & 0,42 & 0,46 & $0,43 \mathrm{~B}$ \\
\hline & Média & $0,42 \mathrm{~b}$ & $0,46 \mathrm{a}$ & $0,46 \mathrm{a}$ & \\
\hline \multirow{4}{*}{$0,20-0,23$} & $\mathrm{RC}$ & 0,40 & 0,38 & 0,43 & $0,41 \mathrm{~A}$ \\
\hline & NT & 0,42 & 0,40 & 0,39 & $0,40 \mathrm{~A}$ \\
\hline & RT & 0,40 & 0,39 & 0,41 & $0,40 \mathrm{~A}$ \\
\hline & Média & $0,40 \mathrm{a}$ & $0,39 \mathrm{a}$ & $0,41 \mathrm{a}$ & \\
\hline & & \multicolumn{4}{|c|}{$\operatorname{MAC}\left(\mathrm{m}^{3} \mathrm{~m}^{-3}\right)$} \\
\hline \multirow{4}{*}{$0,02-0,05$} & $\mathrm{RC}$ & 0,24 & 0,25 & 0,25 & $0,24 \mathrm{~B}$ \\
\hline & NT & 0,32 & 0,34 & 0,31 & $0,32 \mathrm{~A}$ \\
\hline & RT & 0,25 & 0,27 & 0,27 & $0,26 \mathrm{~B}$ \\
\hline & Média & $0,27 \mathrm{a}$ & $0,28 \mathrm{a}$ & $0,28 \mathrm{a}$ & \\
\hline \multirow{4}{*}{$0,10-0,13$} & $\mathrm{RC}$ & $0,12 \mathrm{~A}$ & $0,17 \mathrm{AB}$ & $0,21 \mathrm{~A}$ & $0,18 \mathrm{~B}$ \\
\hline & NT & $0,19 \mathrm{~A}$ & $0,25 \mathrm{~A}$ & $0,20 \mathrm{~A}$ & $0,21 \mathrm{~A}$ \\
\hline & RT & $0,13 \mathrm{~A}$ & $0,15 B$ & $0,19 \mathrm{~A}$ & $0,16 \mathrm{~B}$ \\
\hline & Média & $0,15 b$ & $0,19 a$ & $0,20 \mathrm{a}$ & \\
\hline \multirow{4}{*}{$0,20-0,23$} & $\mathrm{RC}$ & 0,13 & 0,09 & 0,15 & $0,12 \mathrm{~A}$ \\
\hline & NT & 0,13 & 0,12 & 0,11 & $0,12 \mathrm{~A}$ \\
\hline & RT & 0,12 & 0,12 & 0,12 & $0,12 \mathrm{~A}$ \\
\hline & Média & $0,12 \mathrm{a}$ & $0,11 \mathrm{a}$ & $0,13 a$ & \\
\hline \multirow{5}{*}{$0,02-0,05$} & & \multicolumn{4}{|c|}{ MIC $\left(\mathrm{m}^{3} \mathrm{~m}^{-3}\right)$} \\
\hline & $\mathrm{RC}$ & 0,29 & 0,28 & 0,29 & $0,29 \mathrm{~A}$ \\
\hline & NT & 0,27 & 0,24 & 0,25 & $0,25 \mathrm{~B}$ \\
\hline & RT & 0,28 & 0,27 & 0,28 & $0,28 \mathrm{~A}$ \\
\hline & Média & $0,28 \mathrm{a}$ & $0,26 \mathrm{a}$ & $0,27 \mathrm{a}$ & \\
\hline \multirow{4}{*}{$0,10-0,13$} & $\mathrm{RC}$ & 0,28 & 0,38 & 0,27 & $0,31 \mathrm{~A}$ \\
\hline & NT & 0,27 & 0,25 & 0,26 & $0,26 \mathrm{~A}$ \\
\hline & RT & 0,26 & 0,27 & 0,28 & $0,27 \mathrm{~A}$ \\
\hline & Média & $0,27 \mathrm{a}$ & $0,30 \mathrm{a}$ & $0,27 \mathrm{a}$ & \\
\hline \multirow{4}{*}{$0,20-0,23$} & $\mathrm{RC}$ & 0,27 & 0,28 & 0,28 & $0,28 \mathrm{~A}$ \\
\hline & NT & 0,29 & 0,28 & 0,28 & $0,28 \mathrm{~A}$ \\
\hline & RT & 0,27 & 0,28 & 0,30 & $0,28 \mathrm{~A}$ \\
\hline & Média & $0,28 \mathrm{a}$ & $0,28 \mathrm{a}$ & $0,29 a$ & \\
\hline
\end{tabular}

Médias seguidas pela mesma letra maiúscula nas colunas e minúscula nas linhas dentro de cada camada, não diferem significativamente entre si pelo teste de Tukey $(p<0,05)$. A presença ou ausência de letras maiúsculas nas colunas e minúsculas nas linhas, na interação cobertura de inverno versus tráfego, dentro de cada camada, indica interação significativa ou não, respectivamente

de $0,37 \mathrm{~m}^{3} \mathrm{~m}^{-3}$, para ambos os tráfegos sinalizando o incremento de poros no solo, mesmo em maiores profundidades, o que pode ser melhor visualizado na camada de $0,20-0,23 \mathrm{~m}$ a qual teve $\mathrm{Pt}$ maior (em média $0,40 \mathrm{~m}^{3} \mathrm{~m}^{-3}$ ) que aquela encontrada no ano de 2006, em profundidade menor.

Ocorreu interação significativa para a condição de tráfego e cobertura de inverno (NF) para a MAC (Tabela 5), na camada de $0,10-0,13 \mathrm{~m}$. A MAC foi maior $\left(0,25 \mathrm{~m}^{3} \mathrm{~m}^{-3}\right)$ em NT embora sem diferença estatística ao $\mathrm{RC}\left(0,17 \mathrm{~m}^{3} \mathrm{~m}^{-3}\right)$. Para a mesma 
camada, e na média dos valores, a cobertura de solo no inverno influenciou a MAC, sendo menor em AE (Tabela 5). Os valores de MAC estão de acordo com a Ds e Pt. Diminuições da Ds aumentaram a Pt e, em contrapartida, a MAC.

Nas duas primeiras camadas avaliadas a MAC foi maior em solo NT (Tabela 5) na média dos valores. Nesta condição, a MAC foi de $0,32 \mathrm{~m}^{3} \mathrm{~m}^{-3}$ na camada de $0,02-0,05$ contrastando com os $0,21 \mathrm{~m}^{3} \mathrm{~m}^{-3}$ encontrados em 2006 (Debiasi et al., 2008) e de $0,07 \mathrm{~m}^{3} \mathrm{~m}^{-3} \mathrm{em} 2002$ (Beutler, 2005). Nas condições de solo trafegado por trator ou colhedora, a MAC foi maior (Tabela 5) que em $2006\left(0,17 \mathrm{~m}^{3} \mathrm{~m}^{-3}\right)$; na camada de $0,10-0,13 \mathrm{~m}$ a maior $\operatorname{MAC}\left(0,21 \mathrm{~m}^{3} \mathrm{~m}^{-3}\right)$ foi obtida no solo NT, em comparação com a média do solo trafegado $\left(0,17 \mathrm{~m}^{3} \mathrm{~m}^{-3}\right)$; entretanto, nessas duas condições os valores foram maiores do que os obtidos em 2006 (Debiasi et al., 2008), para solo sem e com tráfego, que foram de, respectivamente, 0,11 e $0,10 \mathrm{~m}^{3} \mathrm{~m}^{-3}$. Para a camada de $0,20-$ $0,23 \mathrm{~m}$ os resultados foram semelhantes aos obtidos em 2006 .

Os valores de MIC, semelhantes entre as camadas e os manejos de solo utilizados (Tabela 5), comprovam que o incremento no volume de MAC colaborou para a redução da Ds e no aumento da Pt. A MIC apresentou valores similares aos observados antes da implantação do experimento, em 2002 $\left(0,29 \mathrm{~m}^{3} \mathrm{~m}^{-3}\right)$ (Beutler, 2005) e aos obtidos em $2006\left(0,27 \mathrm{~m}^{3}\right.$ $\left.\mathrm{m}^{-3}\right)$ (Debiasi, 2008).

O presente trabalho contribui para o entendimento do comportamento de parâmetros de qualidade do solo em sistemas conservacionistas e que atuam para a sustentabilidade agrícola. Avaliações no tempo podem indicar mudanças dinâmicas da qualidade física do solo, ocasionadas por práticas integradas de manejo do solo e das culturas.

\section{Conclusões}

1. As práticas integradas de manejo do solo com a semeadura direta, a utilização de plantas de cobertura e o tráfego controlado, são eficazes na recuperação da estrutura física de um solo degradado pela compactação adquirindo, no tempo, qualidade física adequada.

2. O aumento nos teores de matéria orgânica do solo e de suas frações no tempo influencia a diminuição dos valores de densidade e no aumento da macroporosidade e porosidade total do solo.

3. As regiões do solo que, com o tempo, não recebem o tráfego direto dos rodados de máquinas agrícolas têm sua densidade diminuída e porosidade total aumentada.

4. A densidade e a porosidade do solo são sensíveis às mudanças do manejo do solo tal como a fração lábil (particulada) da matéria orgânica do solo sendo considerados, portanto, bons indicadores da qualidade do solo.

\section{Literatura Citada}

Balbinot Júnior, A. A.; Backes, R. L.; Tôrres, A. N. L. Desempenho de plantas invernais na produção de matéria seca e cobertura do solo sob cultivos isolado e em consórcios. Revista de Ciências Agroveterinárias, v.3, p.38-42, 2004.
Bayer, C.; Martin Neto, L.; Mielniczuk, J.; Pavinato, A. Armazenamento de carbono em frações lábeis da matéria orgânica de um Latossolo Vermelho sob plantio direto. Pesquisa Agropecuária Brasileira, v.39, p.677-683, 2004.

Bayer, C.; Mielniczuk, J.; Martin Neto, L. Efeito de sistemas de preparo e de cultura na dinâmica da matéria orgânica e na mitigação das emissões de CO2. Revista Brasileira de Ciência do Solo, v.24, p.599-607, 2000.

Beutler, J. F. Parâmetros de solo e máquinas na semeadura direta de milho e soja em duas coberturas de solo sobre campo natural. Porto Alegre: UFRGS, 2005. 107p. Tese Doutorado

Braida, J. A; Reichert, J. M.; Reinert, D. J.; Sequinatto, L. Elasticidade do solo em função da umidade e do teor de carbono orgânico. Revista Brasileira de Ciência do Solo, v.32, p.477-485, 2008.

Câmara, R. K.; Klein, V. A. Escarificação em plantio direto como técnica de conservação do solo e da água. Revista Brasileira de Ciência do Solo, v.29, p.789-796, 2005.

Conceição, P. C. Agregação e proteção física da matéria orgânica em dois solos do sul do Brasil. Porto Alegre: UFRGS, 138p. 2006. Tese Doutorado

Conceição, P. C.; Amado, T. J. C.; Mielniczuk, J. Qualidade do solo em sistemas de manejo avaliada pela dinâmica da matéria orgânica e atributos relacionados. Revista Brasileira de Ciência do Solo, v.29, p.777-788, 2005.

Debiasi, H. Recuperação física de um Argissolo compactado e suas Implicações sobre o sistema solo-máquina-planta. Porto Alegre: UFRGS, 2008. 263p. Tese Doutorado

Debiasi, H.; Levien, R.; Trein, C. R.; Conte, O.; Mazurana, M. Capacidade de suporte e compressibilidade de um Argissolo, influenciadas pelo tráfego e por plantas de cobertura de inverno. Revista Brasileira de Ciência do Solo, v.32, p.26292637, 2008.

Dexter, A. R. Amelioration of soil by natural processes. Soil and Tillage Research, v. 20, p.87-100, 1991.

Diekow, J.; Mielniczuk, J.; Knicker, H.; Bayer, C; Dick, D. P.; Knabner, I. K. Carbon and nitrogen stocks in physical fractions of a subtropical Acrisol as influenced by long-term no-till cropping systems and $\mathrm{N}$ fertilisation. Plant and Soil, v.268, p.319-328, 2005.

EMBRAPA - Empresa Brasileira de Pesquisa Agropecuária. Centro Nacional de Pesquisa de Solos. Manual de métodos de análise de solos. 2.ed. Rio de Janeiro: EMBRAPA, 1997. 212p.

EMBRAPA - Empresa Brasileira de Pesquisa Agropecuária. Centro Nacional de Pesquisa de Solos. Sistema brasileiro de classificação de solos. 2.ed. Rio de Janeiro: EMBRAPA, 2006. 306p.

Fliebbach, A.; Oberholzer, H. R.; Gunst, L.; Mäder, P. Soil organic matter and biological soil quality indicators after 21 years of organic and conventional farming. Agriculture, Ecosystems \& Environment, v.118, p.273-284, 2007.

Hamza, M. A.; Anderson, W. K. Soil compaction in cropping systems A review of the nature, causes and possible solutions. Soil and Tillage Research, v.82, p.121-145, 2005. 
Jarecki, M. K.; Lal, R.; James, R. Crop management effects on soil carbon sequestration on selected farmers' fields in ortheastern Ohio. Soil and Tillage Research, v.81, p.265276, 2005.

Karlen, D. L.; Mausbach M. J.; Doran J. W.; Cline R. G.; Harris R. F.; Schuman G. E. Soil quality: A concept, definition, and framework for evaluation. Soil Science Society of America Journal, v.61, p.4-10, 1997.

Oades, J. M. The role of biology in the formation, stabilization and degradation of soil structure. Geoderma, v.56, p.377400, 1993.

Tedesco, M. J.; Gianello, C. Bissani, C. A.; Bohnen, H.; Volkweiss, S. J. Análise de solo, plantas e outros materiais. 2.ed. Porto Alegre: UFRGS, 1995. 174p.

Peth, S.; Horn, R. The mechanical behavior of structured and homogenized soil under repeated loading. Journal of Plant Nutrition and Soil Science, v.169, p.401-410, 2006.

Reichert, J. M.; Reinert, D. J.; Braida, J. A. Qualidade dos solos e sustentabilidade de sistemas agrícolas. Revista Ciência \& Ambiente, v.27, p.29-48, 2003.
SAS Institute. Getting started with the SAS learning edition. Cary: SAS, 2002. 200p.

Sequinatto, L. Qualidade física do solo e rendimento de grãos num Argissolo em processo de recuperação. Porto Alegre: UFRGS, 2010. 141p. Tese Doutorado

Soane, B. D. The role of organic matter in soil compactability: a review of some practical aspects. Soil and Tillage Research, v.16, p.179-201, 1990.

Souza, E. D.; Costa, S. E. V. G. A.; Anghinoni, I.; Carvalho, P. C. F.; Andrigueti, M.; Cao, E. Estoques de carbono orgânico e de nitrogênio no solo em sistema de integração lavoura-pecuária em plantio direto, submetido a intensidades de pastejo. Revista Brasileira de Ciência do Solo, v.33, p.1829-1836, 2009.

Veiga, M.; Horn, R.; Reinert. D. J.; Reichert, J. M. Soil compressibility and penetrability of an Oxisol from southern Brazil, as affected by long-term tillage systems. Soil and Tillage Research, v.92, p.104-113, 2007.

Vezzani, F. M.; Mielniczuk, J. Agregação e estoque de carbono em Argissolo submetido a diferentes práticas de manejo agrícola. Revista Brasileira de Ciência do Solo, v.35, p.213223, 2011 